\title{
Analysis on the Design and Production of Special Effects for 3D Games
}

\author{
Yuan Yang \\ College of Sino-Korea Multimedia Design \\ Shanghai University of Engineering Science \\ Shanghai, China \\ e-mail: yoyo810608@163.com
}

\author{
zhouyun Kang \\ College of Sino-Korea Multimedia Design \\ Shanghai University of Engineering Science \\ Shanghai, China
}

\begin{abstract}
The special effects create lifelike images for three dimension virtual games, and serve as important elements for the overall setting and stimulation of the real. By introducing different types of special effects, and how these effects are created, this article presents a systematic illustration of the very importance of special effects design for games.
\end{abstract}

\section{Keywords—game effects; Particle effects; texture effects}

\section{INTRODUCTION}

Special effects are mainly used by entertainment industries for visual attraction, which obtained it's wider application over motion pictures, animations, games and virtual reality. The design of various types of special effects bring joy to the audience, at the same time, excites our imagination with the creation of wonderlands and battle fields. The use of special effects adds both realistic and artistic features for the games, in which way provides the players with upgraded experience of immersion.

Not only supports the visual effects, special effects are also crucial for the game-play itself. With the help of special effects, plays can perform different skills and use every kind of props to change the environment for interaction and combat. Besides making the realistic interaction between players become possible, scenes like raining, snowing and fog cue created by special effects are vital for the foiling the entire setting of any certain games. The conception, reality and texture brought by special effects came from a combination of philosophic ideas, physics, and aesthetics, construct scenarios that challenges our imagination. Based on realistic and logical concepts and limited imagination, special effect conveys a broader and more vivid virtual world.

\section{The CONSTITUTION OF 3D SPECIAL EFFECTS FOR GAMES}

As part of game art, special effects can draw attentions of the players as well as actual sales promotion. The feeling of fulfillment brought by special effects to the players and the nirvana as they called it, are what they enjoy to relish.

Divided by features of the objects, there are three types of different special effects for 3D games: 1. Light(optical)effect, which is most commonly used. Mostly appear as texture effects. Light effects in games include spot and condensation of lights, as well as light in different shapes or transforming lights. spotlights often appear as condensation in the games, in which lights can also be programmed into different shapes for requirements of the special effects. Transforming lights often appear as luminous words, lightening or flames. For example, plug-ins that create shining sharp blades can map out a certain locus, which can be very useful when building scenes for combats with knife and swords. 2. Besides optical effect, smoke and fog are also commonly applied as special effects. Similar to creating lights, fog and smoke are produced by patch stimulation. For instance, when characters in the games jump around the scene, fog cue ought to appeared immediately.3. The third type of special effect comes with the trait and texture changes of different materials and objects, waterfall, flowing magma, or a glowing robot. Lastly, the production of complicated effects like explosion or Tyndall Phenomena, are combination of multiple techniques, which can also produce special effects like fireworks.

The South Korean game Blades and Souls is the representation of well-designed optical effects, the special effects make the image and setting more vivid and lifelike, especially when the subjects, surrounding, atmosphere and other elements interact with each other, the moving waves and different shades over muscles are reasonable and excellent example to illustrate that.

\section{THE IMPLEMENTATION OF GAME EFFECTS}

Special effects in $3 \mathrm{D}$ contribute to the dynamic lighting in three dimensional games, mainly include particle animation effects, simulative animation effects, mapping animation effects and mixed effects, among which particle effects are most widely used. Particle effect can help to achieve the majority of special effects, which elevate efficiency and creates marvelous out-comes. Particle system is the kinetic systems of special effects for $3 \mathrm{D}$ software, which can accomplish the stimulation of various natural phenomenon like dust, flames, meteor, lightening, clouds, rain and snow, blood or waterfall. Stimulation effects are usually presented in 3D among games, which can be observed from different angles. The advantage of stimulation effects lies in the recognition of it, which might leave strong 
impression to the players. Mapping effects usually came as a series of sequenced frames of animation that played according to this order. It can create a relevantly rich range of effects with simpler technology and limited resources. Mixed effect combines particle effects and stimulation effects, which is also quite common during the design of special effects. The production and implementation of special effects are usually created by the effects editors in the game engine, so the exact procedures of special effect production may vary from different modes of editors used by different game companies.

Particle effects are mostly realized in the effects editors, such as fireworks through special effects, which requires a thorough analysis of the shape, color and moving traits of fireworks. The features concluded from the analysis will later be represented by certain types of particles. Particle animation effects are built by particle systems, which serves as a process model than combines the shape and the animation together. As individuals in a completed modeling, every particle has it's own life cycle, and the generation of particles is also random. The size, texture, transparency, speed and direction, or the life cycle of each particle should be specified based on the shared features of different groups of particles as designed.

The mapping material springing from the ideas of the designers are crucial for the accuracy of stimulating reality during the production of $3 \mathrm{D}$ special effects. The way actual object moves in natural environment should be closely studied and followed, which is the basic principle of presenting lifelike effects in the $3 \mathrm{D}$ games.

\section{THE DESIGN AND REQUIREMENTS OF PRODUCING SPECIAL EFFECTS}

The production of special effects is usually conducted by effect editors in the game engine, which is based on the compact of 2D and 3D designing. The maps and models can be created using 2D or 3D software, 3D Max is the most commonly used one among them. Operators should be equipped with not only necessary skill of 3D Max, but also profound knowledge about mirroring, reflection and duplication of 3D modeling, as well as the skills to edit and adjust the color, texture, shape and movements of exogenous materials needed in the production, in order to bring out the ultimate effects as anticipated.

\section{A. Production of special effects for game props}

Before getting into detailed production, the target of the production needs to be clarified. The same as other elements of designing, for example, blades of swords and knives, the design draft of each prop should be determined first, matching appropriate shades and shapes to the blade. Following with the modeling and mapping of the props in 3D software such as 3DMAX. The mapping should proceed according to the original map, the material can be self-made or use existing resource, the importance is to imitate the color and effects of real blades. To complete the production of special effects, A plug-in called GhostTrals can be effectively used. editing and adjusting statics of shades from different parts of the blade in the software, and emphasizing certain effects by using a certain color or transparent texture of maps. The relevant files be copied into the game engine for rending should be the final step.

Detailed production process is as follows: Design appropriate color and shape for blade when a clear game theme and game style are in place. The game discussed here has a Diablo realistic style, therefore to give the props and characters a matching effect, blue and purple are chosen as the primary color. "Fig. 1" is the two-dimensional sketch of blade lighting.

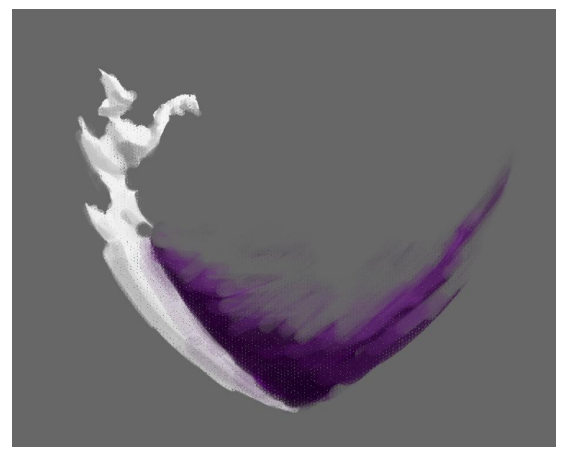

Fig. 1. Blade Lighting Special Effect - Two-dimensional Sketch

Complete modeling in three-dimensional software while using two-dimensional design as the base. "Fig. 2" and "Fig. 3 " shows the blade and mapping/texture? effect in 3DMAX.

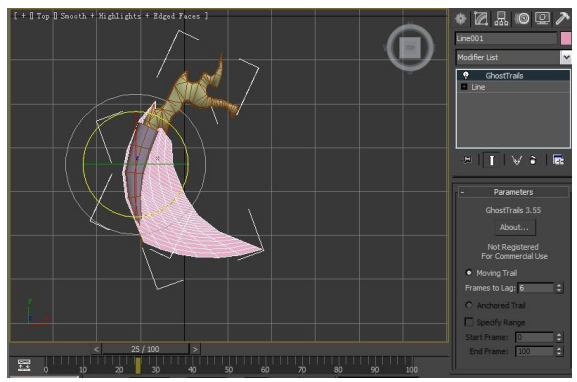

Fig. 2. Blade Lighting Using Plug-in "GhostTrals"

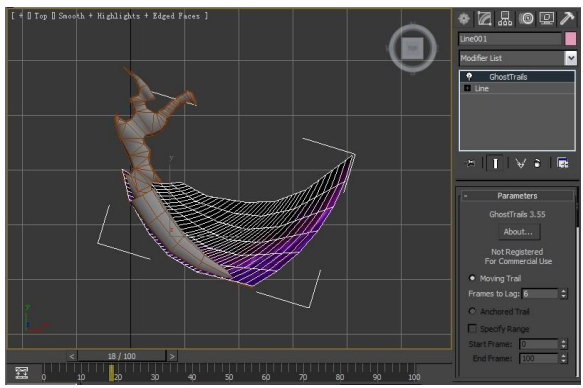

Fig. 3. Mapping Effect

Below shows the mapping effect in 3DMAX after completing the production and mapping of blade lighting. During the production process, be careful with adjusting the display and brightness of the maps "Fig. 4". 


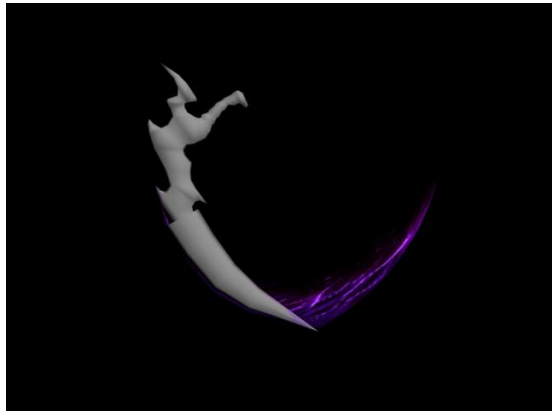

Fig. 4. Effect after Real-time Rendering

The final step is to perfect weapon modelling as well as complete the final rendering effect using UDK game engine "fig. 5" \& "Fig. 6".
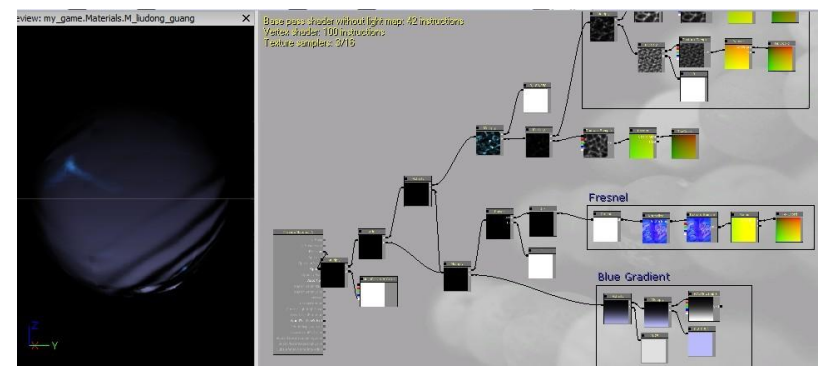

Fig. 5. Special Effect Maps in UDK Engine

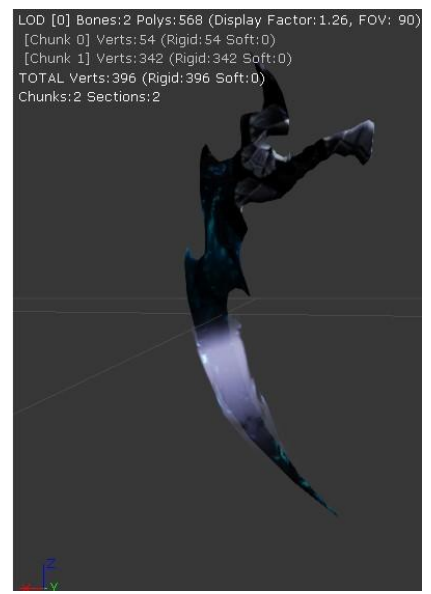

Fig. 6. Final Rendering Effect for Weapon

\section{B. Special effects production for characters}

The special effects production for characters is relevantly simple that doesn't require much detailed work, for example the Pixar animation Brave, the company used three years for creating the special effects of the main character's red hair, for which Pixar even remodeled it's producing system for hair and fur.

For characters in the games, special effects are used to represent features of their clothing and movements, the movements during combats are designed and roughly structured, later achieved with the help of special effects for instance. Most of them are created by mapping effects because they took up a smaller portion of the RAM which better insures the operation of the whole system.

Effects for the characters usually real the realistic features and texture, the keynote of the character should be firstly schemed while planing, then the unpredictable atmosphere will be further achieved by special effects.the key is to find out the UV mapping materials that matches the characteristics of different rules. UV material helps to produce natural effects and saves RAM. Models of skin (covering)should be produced while rendering the stimulation, in order to assure the consistency of movements. To map out the traits in the image as designed, programmers need to add extra mapping files to the texture editor as well as adjust the UV movement setting while producing, in this way, the special effects production for certain characters is accomplished. Simple animation techniques can be attached to the models of special effects for characters, to synchronize the movement of special effects with movements of the characters.

Detailed special-effect production process for characters is as follows: First finalize the materials that show features of the character, then finalize character modeling and mapping as well as producing a copy of skin model as shown in "Fig. 7" below.

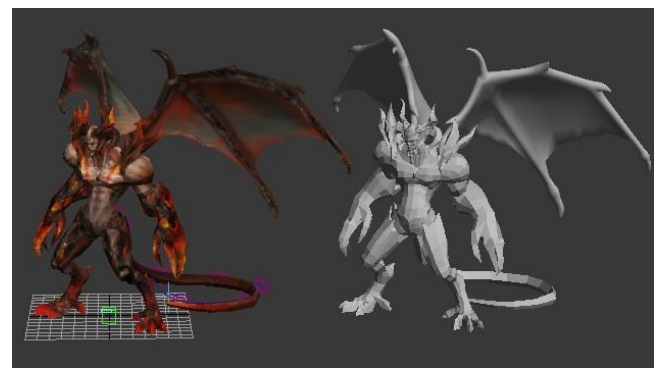

Fig. 7. Character Model and Skin Model

Secondly, as shown in "Fig. 8", add maps in the material editing tool and copy the example to Opacity and Color; select Color. Although the sphere map does not resemble the previous example, after a few simple settings, objects will be in motion.

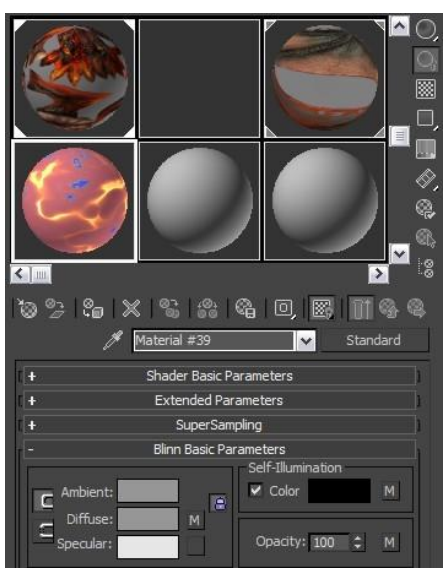

Fig. 8. Adding Maps in the Material Editing Tool 
The next step is as shown in "Fig. 9". Open Extended Parameter in the Material Editing Tool, change RGB Intensity to Alpha.
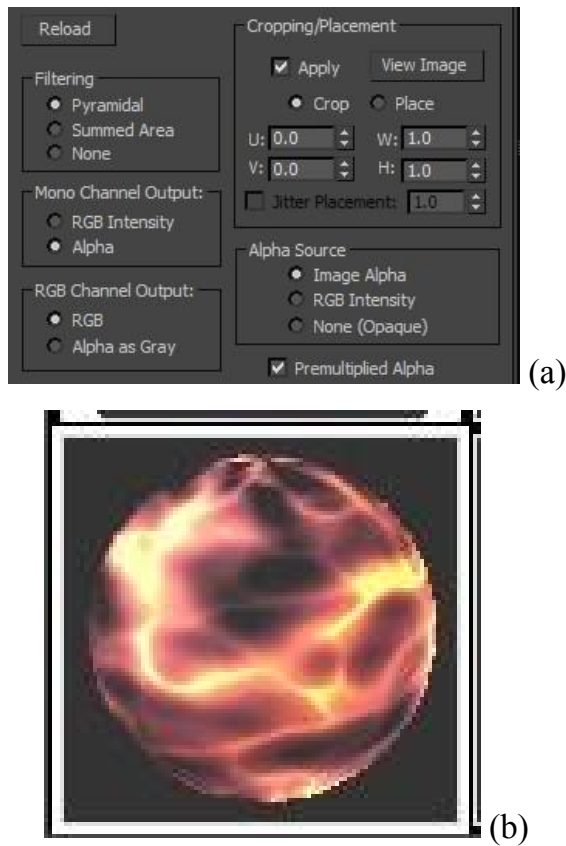

Fig. 9. Extended Parameters Setting
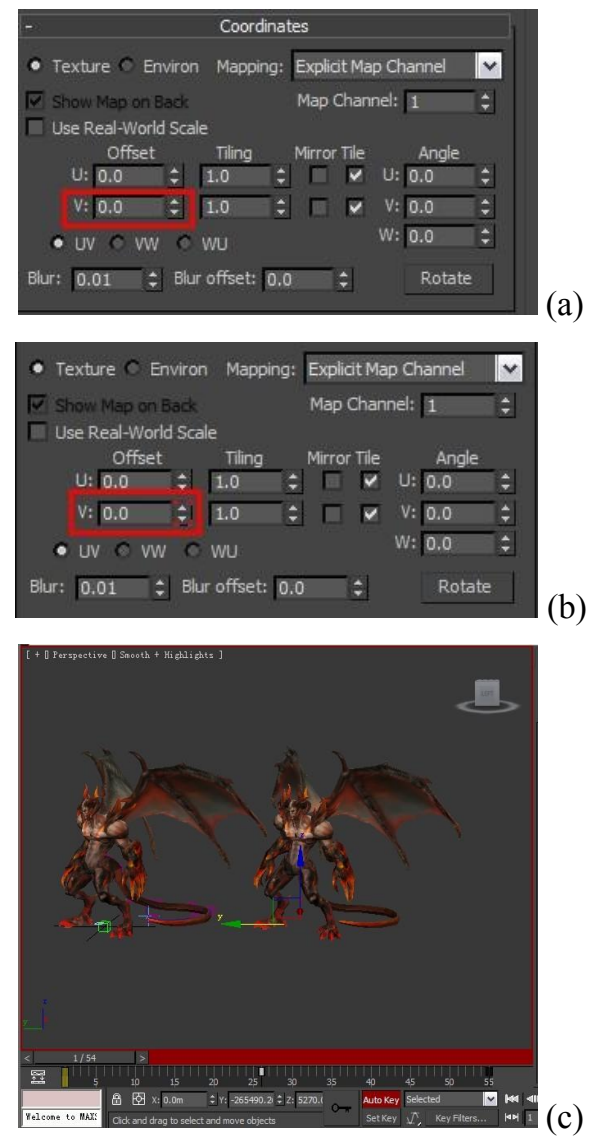

Fig. 10. UV Motion Setting
$\mathrm{V}$ value shown in "Fig 10 (a)" is the key to achieve sphere movement. Opens Auto Key in software, adjust the frames to the first one, set the value of $\mathrm{V}$ to 0 , and then pull all the actions of the last frame, set a value of $\mathrm{V}$ to 1.0 , turn off Auto Key. Now we shall see a red line/frame appear in the drop-down of $\mathrm{V}$ value as shown in "Fig. 10 (b)". When pulling again around the left and right frames, $\mathrm{V}$ value will automatically change. Thus the trajectory map is complete.

Finally, match the finished character map to the model: the model has a translucent effect, and the character has noticeable waving effects all around its body. Until now, special effect for character is primarily completed. References see "Fig 11 (a), (b), (c)" below.

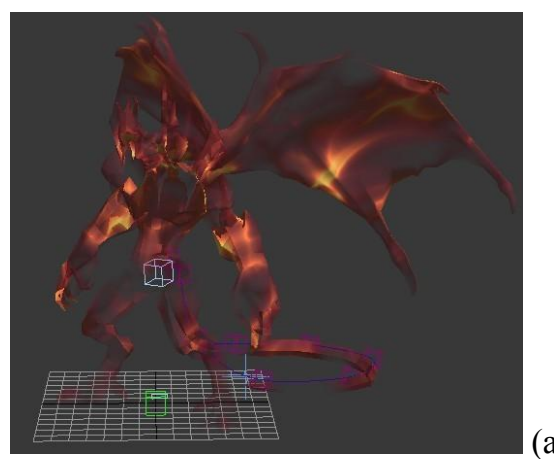

(a)
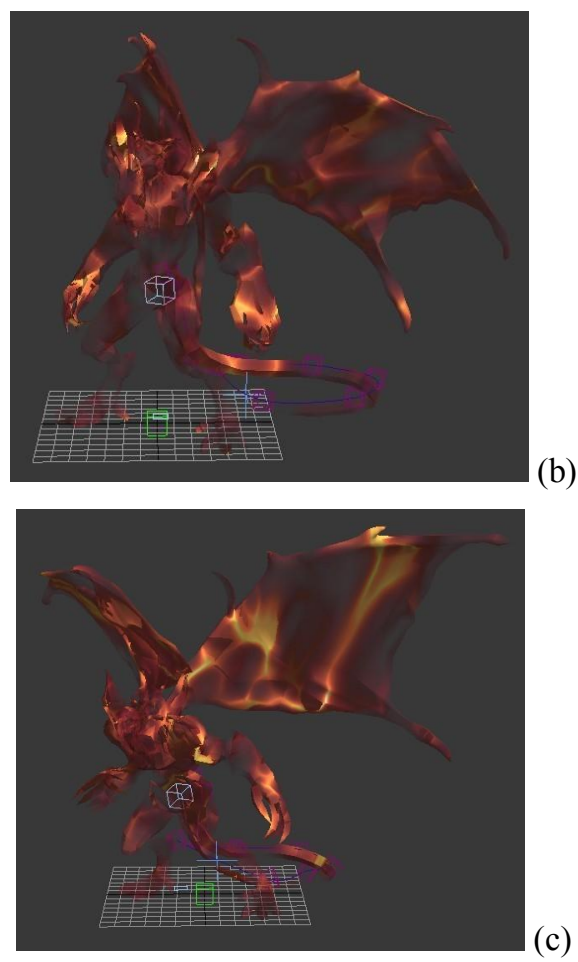

Fig. 11. FSpecial Effect Character Mapping

\section{Production of Special effects for Game environment}

Game environment includes the scene, natural background of the games, in which special effects are widely used. Currently, most well-made game setting are produced by the Normal Mapping Technology, to stimulate the details on the surface of irregular subjects, like wave motion, ruins 
of brick walls. this technology can promote efficiency while assuring high quality of special effects, the creation of other effects like fog, rain and snow, flames or explosion are also assisted by particle systems.

\section{CONCLUSION}

In summary, the design of special effects for games is built on computer technology, both hardware and software. It's the assemble of computer engineering, aesthetics, literature, philosophy and arts. Special effect requires the accurate stimulation of the reality, as the changing of $3 \mathrm{D}$ digital production, visual reality became technical focus of the digital entertainment industry, the same as film effects, the designers aim at creating better and more realistic images with improving texture. Designing special effects for games elevates the atmosphere and setting, also improves the overall quality of the games and sense of immersion for the players.

\section{REFERENCES}

[1] Zhao Ming, "Reasearch and Implement of the Special Effect in 3D Game by Using DirectX,"A Dissertation for the Degree of M.Eng, pp. 19-22, January,2009

[2] Zhang YunYuan, "Review technology special effects for online Games," Fujian Computer. September,201 